Recebido em 05/2017. Aceito para publicação em 11/2018.

\title{
CÁLCULO DO DESLOCAMENTO DE UMA BARRA RíGIDA SUSPENSA CONSTRUÍDA DE DIVERSOS MATERIAIS QUANDO UMA FORÇA É APLICADA UTILIZANDO O SCILAB
}

\section{CALCULATION OF THE DISPLACEMENT OF A SUSPENDED RIGID BAR CONSTRUCTED FROM DIFFERENT MATERIALS WHEN A FORCE IS APPLIED USING SCILAB}

\author{
Luciana Claudia Martins Ferreira Diogenes ${ }^{1}$
}

\begin{abstract}
Resumo: Em projetos de engenharia que envolvem estudo ou construção de máquinas, edificações ou outros sistemas, devem ter as suas estruturas dimensionadas e construídas de materiais resistentes para suportar as cargas que agem sobre elas. O objeto de estudo neste trabalho consiste de um sistema constituído de uma barra rígida suspensa em equilíbrio estático com dois cilindros deformáveis sob ação de um carregamento de $30 \mathrm{kN}$ em um único ponto. Todo o estudo é considerado na região elástica de escoamento. Enquanto um dos cilindros tem o seu material bem definido, sendo constituído de alumínio, no outro, o material é substituído por diversas vezes para se conhecer qual material de uma lista proposta não poderia suportar tal carregamento. Com a ajuda do software Scilab, pode-se construir um código que permite substituir a propriedade dos materiais conhecida como módulo de elasticidade nos cálculos para se obter os deslocamentos provocados pela carga e, também, saber se o material tem a sua tensão dentro do limite da tensão de escoamento, na região elástica, onde os cálculos são válidos.
\end{abstract}

Palavras-chave: Barra rígida; deslocamento; estática; força axial; resistência dos materiais.

Abstract: In engineering projects involving the study or construction of machines, buildings or other systems, their structures must be dimensioned and constructed using materials that are resistant to withstand the loads acting on them. The object of study in this work consists of a system composed of a suspended rigid rod in static equilibrium with two deformable cylinders under a load of $30 \mathrm{kN}$ in a single point. The entire study is considered in the elastic region of flow. While one of the cylinders has its material well defined, being made of aluminum, in the other, the material is replaced several times to know which material from a proposed list could not withstand such loading. With the help of the Scilab software, one can construct a code that allows the substitution of the property of the materials known as modulus of elasticity in the calculations to obtain the displacements caused by the load and also to know if the material has its tension within the limit of the yield strength in the elastic region, where the calculations are valid.

Keywords: Rigid rod; displacement; statics; axial force; strength of materials.

\section{INTRODUÇÃO}

A mecânica é a ciência que lida com os efeitos da força interagindo sobre os objetos, que podem ser partículas our corpos rígidos e pode ser dividida em duas partes: a estática e a dinâmica, sendo que a estática lida com equilíbrio de corpos e a dinâmica, lida com o movimento dos corpos. Um corpo rígido é definido quando se é considerada

\footnotetext{
1 Doutorado em Engenharia Mecânica, Universidade Estadual de Campinas - Unicamp, Brasil. E-mail: lucianafem@yahoo.com.br.
} 
desprezível a variação da distância entre dois pontos quaisquer do objeto (MERIAM; KRAIGE, 2009).

Para (HIBBELER, 2005, p. 1), o ramo da mecânica que estuda as relações entre as cargas externas submetidas a um corpo deformável e a intensidade das forças internas que atuam nele é chamado de resistência dos materiais. Nesse ramo, também se realiza o cálculo das deformações do corpo e se estuda a sua estabilidade quando sujeito a forças externas. Em qualquer projeto envolvendo estruturas, é necessário usar os princípios da estática para determinar as forças que agem sobre os vários elementos, bem como no seu interior.

O diagrama de corpo livre é um esboço de todas as forças que atuam sobre o ponto material. Para traçar o diagrama do corpo livre, três passos podem ser considerados (HIBBELER, 2005, p. 70):

1) Desenhar o contorno do ponto material como sendo livre de seu entorno;

2) Mostrar todas as forças que atuam sobre o ponto material; e

3) Identificar cada força conhecida, anotando suas intensidades, direções e sentidos.

Após traçar o diagrama de forças em um sistema em equilíbrio estático e sujeito a um sistema de forças no plano $x-y$, as condições de equilíbrio, em duas dimensões, são escritas da seguinte maneira (HIBBELER, 2005, p. 70):

$$
\begin{aligned}
\sum \boldsymbol{F}_{\boldsymbol{x}} & =\mathbf{0} \\
\sum \boldsymbol{F}_{\boldsymbol{y}} & =\mathbf{0} \\
\sum \boldsymbol{M}_{\mathbf{0}} & =\mathbf{0}
\end{aligned}
$$

onde $F_{x}$ e $F_{y}$ são as forças que atuam nos eixos $x$ e $y$, respectivamente e $\sum M_{0}$ representa a soma dos momento binários e dos momentos de todos os componentes de força em relação a um eixo perpendicular ao plano $x-y$, passando pelo ponto $O$, dentro ou fora do corpo.

O cálculo do deslocamento $\boldsymbol{\delta}$ em uma barra homogênea de comprimento $L$ e secção transversal A, provocado por um carregamento axial de força $\mathrm{P}$, em uma de suas extremidades (figura 1), pode ser obtido a partir das equações de tensão e deformação.Se o estudo está sendo realizado na região do limite de proporcionalidade (a tensão $\sigma$ deverá ser menor que a tensão de escoamento $\sigma_{E}$ ), pode-se usar a relação (BEER et al.,2008, p. 129): 


$$
\sigma=E \epsilon
$$

onde $E$ é o módulo de elasticidade e $\varepsilon$ é a deformação.

Sabendo que a tensão $\sigma$ é dada pela razão da força sobre área, a deformação $\varepsilon$ pode ser obtida como (LEET; UANG; GILBERT, 2010, p. 27; NASH; POTTER, 2014, p. 2), tem-se:

$$
\epsilon=\frac{\sigma}{E}=\frac{P}{A E}
$$

A deformação também é obtida pela seguinte equação:

$$
\epsilon=\frac{\delta}{L}
$$

onde $\boldsymbol{\delta}$ é o deslocamento.

Dessa forma, o deslocamento provocado por um carregamento axial é dado por:

$$
\delta=\frac{P L}{A E}
$$

Figura 1 - Barra de comprimento $L$ sobre um carregamento axial $P$ em uma de suas extremidades.

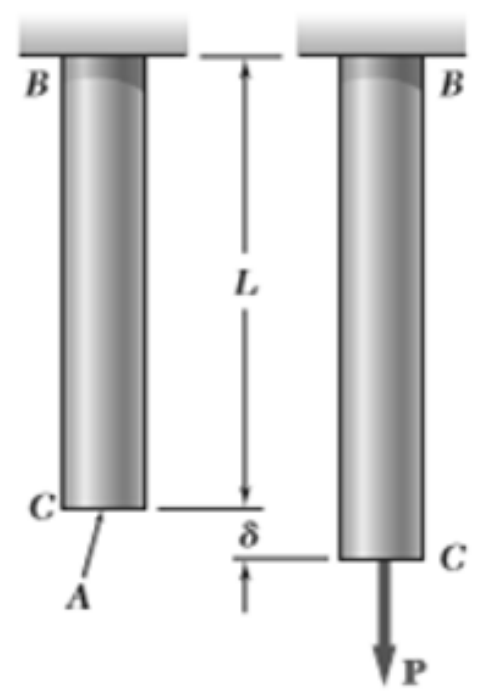

Fonte: (BEER et al., 2008, p. 81).

Segundo Beer et al. (2008), quando existem adicionais suportes para manter um corpo em equilíbrio, as reações tornam-se desconhecidas quando utilizada as equações de equilíbrio. Uma técnica para resolver casos como esse é utilizar o método da superposição. Tal método consiste em escolher uma das reações redundantes e, a partir daí, eliminar o seu suporte. A solução para esse problema é encontrada somando-se os 
resultados das deformações provocadas pelas forças e pela reação redundante.

$\mathrm{O}$ quadro 1 mostra valores de tensão de escoamento $\sigma_{\mathrm{E}}$ e de módulo de elasticidade $E$ de alguns materiais que irão constituir o cilindro de material variável. $A$ tensão de escoamento será comparada com a tensão calculada para saber se o sistema está operando dentro do regime de elasticidade. Caso contrário, os cálculos de deslocamento serão desprezados. O módulo de eslasticidade será útil para o cálculo do deslocamento, conforme mostrado na eq. 7.

Quadro 1 - Tensão de escoamento e módulo de elasticidade para alguns materiais.

\begin{tabular}{|c|c|c|}
\hline Material & $\begin{array}{c}\text { Tensão de } \\
\text { escoamento } \sigma_{E} \\
(\mathrm{MPa})\end{array}$ & $\begin{array}{c}\text { Módulo de } \\
\text { elasticidade } \\
\text { (GPa) }\end{array}$ \\
\hline Aço estrutural (ASTM-A36) & 250 & 200 \\
\hline $\begin{array}{l}\text { Aço baixa liga e alta resistência } \\
\text { (ASTM-A709 Classe } 345 \text { ) }\end{array}$ & 0 & 200 \\
\hline $\begin{array}{l}\text { Aço baixa liga e alta resistência } \\
\text { (ASTM-A913 Classe 450) }\end{array}$ & 0 & 200 \\
\hline $\begin{array}{l}\text { Aço baixa liga e alta resistência } \\
\text { (ASTM-A992 Classe } 345 \text { ) }\end{array}$ & 0 & 200 \\
\hline $\begin{array}{l}\text { Aço temperado e revenido ASTM- } \\
\text { A709 CLASSE } 690\end{array}$ & 0 & 200 \\
\hline $\begin{array}{l}\text { Aço inoxidável AISI } 302 \text { laminado a } \\
\text { frio }\end{array}$ & 0 & 190 \\
\hline Aço inoxidável AISI 302 recozido & 0 & 190 \\
\hline Aço de reforço média resistência & 77 & 0 \\
\hline Aço de reforço alta resistência & 77 & 0 \\
\hline $\begin{array}{l}\text { Ferro fundido cinzento } 4,5 \% \text { C, } \\
\text { ASTM A-48 }\end{array}$ & 0 & 69 \\
\hline $\begin{array}{l}\text { Ferro fundido maleável } 2 \% \mathrm{C}, 1 \% \mathrm{Si} \text {, } \\
\text { ASTM A-47 }\end{array}$ & 230 & 165 \\
\hline Alumínio liga $1100-\mathrm{H} 14(99 \% \mathrm{Al})$ & 95 & 70 \\
\hline Alumínio liga 2012-T6 & 400 & 75 \\
\hline Alumínio liga 2024-T4 & 325 & 73 \\
\hline Alumínio liga 5456-H116 & 230 & 72 \\
\hline Alumínio liga 6061-T6 & 240 & 70 \\
\hline
\end{tabular}




\begin{tabular}{|c|c|c|}
\hline Alumínio liga 7075-T6 & 500 & 72 \\
\hline $\begin{array}{l}\text { Cobre livre de oxigênio }(99,9 \% \mathrm{Cu}) \\
\text { recozido }\end{array}$ & 70 & 120 \\
\hline $\begin{array}{l}\text { Cobre livre de oxigênio }(99,9 \% \mathrm{Cu}) \\
\text { trefilado a frio }\end{array}$ & 265 & 120 \\
\hline $\begin{array}{l}\text { Latão amarelo }(65 \% \mathrm{Cu}, 35 \% \mathrm{Zn}) \\
\text { laminado a frio }\end{array}$ & 410 & 105 \\
\hline $\begin{array}{l}\text { Latão amarelo }(65 \% \mathrm{Cu}, 35 \% \mathrm{Zn}) \\
\text { recozido }\end{array}$ & 100 & 105 \\
\hline $\begin{array}{l}\text { Latão vermelho }(85 \% \mathrm{Cu}, 15 \% \mathrm{Zn}) \\
\text { laminado a frio }\end{array}$ & 435 & 120 \\
\hline $\begin{array}{l}\text { Latão vermelho }(85 \% \mathrm{Cu}, 15 \% \mathrm{Zn}) \\
\text { recozido }\end{array}$ & 70 & 120 \\
\hline $\begin{array}{l}\text { Liga bronze + estanho (88\% Cu, } 8 \% \\
\text { Sn, } 4 \% \mathrm{Zn})\end{array}$ & 145 & 95 \\
\hline $\begin{array}{l}\text { Liga bronze + manganês (63\% Cu, } \\
25 \% \mathrm{Zn}, 6 \% \mathrm{Al}, 3 \% \mathrm{Mn}, 3 \% \mathrm{Fe})\end{array}$ & 330 & 105 \\
\hline $\begin{array}{l}\text { Liga bronze + alumínio (81\% Cu, 4\% } \\
\mathrm{Ni}, 4 \% \mathrm{Fe}, 11 \% \mathrm{Al})\end{array}$ & 275 & 110 \\
\hline
\end{tabular}

Fonte: (BEER et al., 2008, p. 766).

O Scilab é um software gratuito e disponível para download na internet aplicado à computação numérica (DE OLIVEIRA; DE OLIVEIRA; BUENO, 2016). Esse software será utilizado, neste trabalho, para calcular os deslocamentos provocados pela carga uniaxial e os valores de tensão quando um dos cilindros tiver seu material substituído por um dos materiais listados na tabela 01. Dessa forma, construindo-se um código, fica mais fácil estudar o sistema proposto.

\section{METODOLOGIA}

O sistema estudado é constituído por uma barra rígida $B D E$, um cilindro $A B$ e um cilindro CD (figura 2), sendo que este tem o material alterado de acordo com a tabela 1. As dimensões da barra, dos cilindros, módulo de elasticidade e força aplicada no ponto A, são:

- Comprimento $\mathrm{x}_{\mathrm{BD}}$ da barra rígida, $\mathrm{x}_{\mathrm{BD}}=0,2 \mathrm{~m}$; 
- Comprimento $\mathrm{X}_{\mathrm{DE}}$ da barra rígida, $\mathrm{X}_{\mathrm{DE}}=0,4 \mathrm{~m}$;

- Altura do cilindro $A B, y_{A B}=0,3 \mathrm{~m}$;

- Altura do cilindro $B C, \mathrm{y}_{B C}=0,4 \mathrm{~m}$;

- Área do cilindro $A B, A_{A B}=500 \mathrm{~mm}^{2}$;

- Área do cilindro $C D, A_{C D}=600 \mathrm{~mm}^{2}$;

- Força $\mathrm{P}_{\mathrm{E}}=30 \mathrm{kN}$;

- Módulo de elasticidade do cilindro $A B, E_{A B}=70 \mathrm{GPa}$; e

- Módulo de elasticidade do cilindro $C D, E_{C D}=$ veja tabela 1 .

Figura 2 - Barra rígida BDE suspensa pelos cilindros $A B$ de alumínio e $C D$ de matérias diferentes com as indicações das variáveis de comprimento e altura e do ponto de aplicação de força.

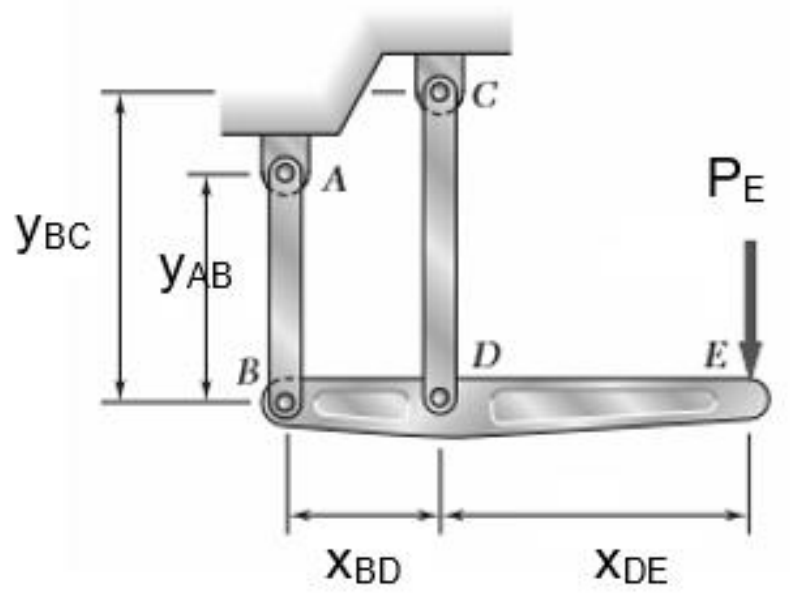

Fonte: (BEER et al., 2008, p. 83).

Para se calcular as forças $F_{C D}$ e $F_{A B}$, é necessário desenhar o diagrama de corpo livre da barra rígida BDE (figura 3). 
Figura 3 - Diagrama de corpo livre da barra BDE.

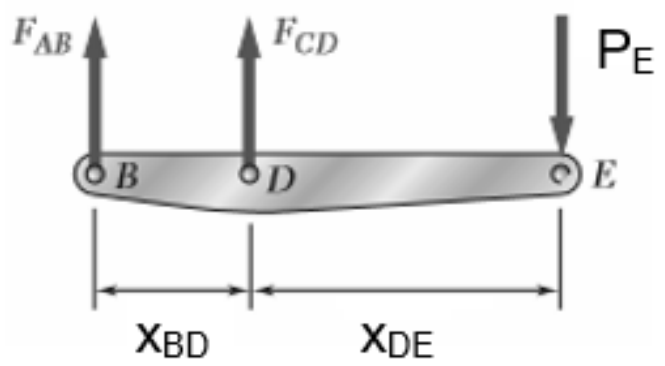

Fonte: (BEER et al., 2008, p. 83).

De acordo com a eq. 3, a equação de equilíbrio para o momento no ponto B pode ser escrita como:

$$
\begin{gathered}
-P_{E}\left(x_{B D}+x_{D E}\right)+F_{C D} x_{B D}=0 \\
F_{C D}=P_{E} \frac{\left(x_{B D}+x_{D E}\right)}{x_{B D}}
\end{gathered}
$$

No ponto $D$, a equação retratada sobre o equilíbrio do momento é dada por:

$$
\begin{gathered}
-P_{E} x_{D E}-F_{A B} x_{B D}=0 \\
F_{A B}=-P_{E} \frac{x_{D E}}{x_{B D}}
\end{gathered}
$$

As forças internas atuantes em cada cilindro de sustentação são de compressão, no cilindro $A B$ e de tração no cilindro $B C$, devido aos sinais negativo e positivo, respectivamente. As forças são visualizadas na figura 4.

Figura 4 - Em a) mostram as forças de compressão na barra $A B$ e em b) as forças de tração na barra CD.
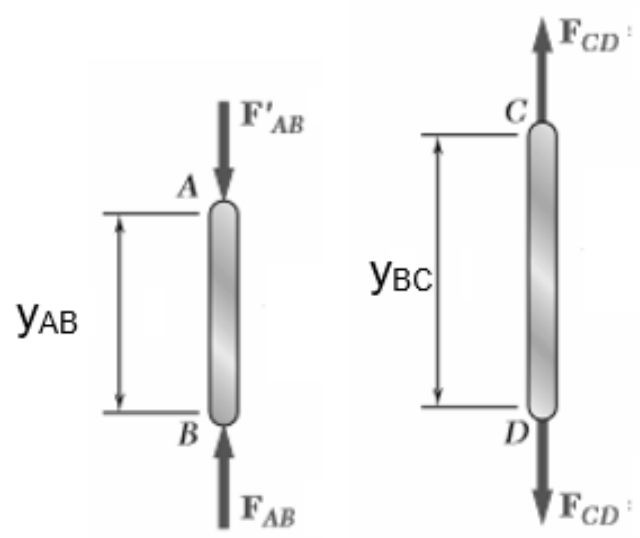

Fonte: (BEER et al., 2008, p. 83).

Ao se aplicar uma força no ponto $\mathrm{E}$, a barra rígida é deslocada de acordo com a 
figura 5. Conforme a equação 7 , no ponto $B$, o deslocamento $\delta_{B}$ é calculado como:

$$
\delta_{B}=\frac{F_{A B} y_{A B}}{A_{B D} E_{B D}}
$$

No ponto $D$, o deslocamento $\delta_{D}$ é calculado como:

$$
\delta_{D}=\frac{F_{C D} y_{B C}}{A_{C D} E_{C D}}
$$

sendo que o valor de $E_{C D}$ é variável devido à troca de material que constitui o cilindro CD.

Figura 5 - Deslocamento B'D’E' da barra rígida BDE.

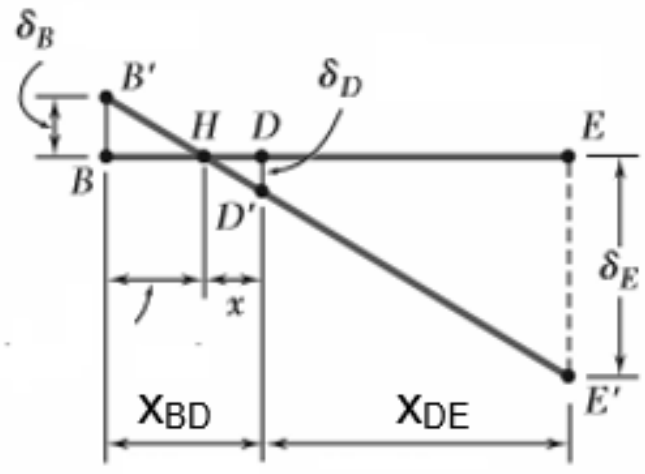

Fonte: (BEER et al., 2008, p. 83).

Observando-se a figura 5, por semelhança de triângulos, pode-se encontrar o valor de $\mathrm{x}$ pela equação 12 :

$$
\begin{gathered}
\frac{B B^{\prime}}{D D^{\prime}}=\frac{B H}{H D} \\
\frac{\left|\delta_{B}\right|}{\delta_{D}}=\frac{x_{B D}-x}{x} \\
x=\frac{\delta_{D} x_{B D}}{\delta_{D}+\left|\delta_{B}\right|}
\end{gathered}
$$

O deslocamento $\delta_{E}$ é encontrado pela equação 13:

$$
\frac{E E^{\prime}}{D D^{\prime}}=\frac{H E}{H D}
$$




$$
\begin{gathered}
\frac{\delta_{E}}{\delta_{D}}=\frac{x_{B D}+x}{x} \\
\delta_{E}=\delta_{D} \frac{x_{D E+} x}{x}
\end{gathered}
$$

Para encontrar os valores das forças $F_{C D}$ e $F_{A B}$, o software Scilab é utilizado e os cálculos são apresentados na seção dos resultados.

Os valores de deslocamento $\delta_{B}, \delta_{D}$ e $\delta_{E}$ são encontrados posteriormente ao cálculo das forças.

\section{RESULTADOS}

O quadro 2 mostra o resultado das forças FCE e FDF obtidos por intermédio da simulação no Scilab, para cada material listado no quadro 1.

O quadro 3 mostra o cálculo de tensão $\sigma_{C D}$ e o compara com a tensão de escoamento $\sigma_{\mathrm{eCD}}$. Se $\sigma_{\mathrm{eCD}}<\sigma_{\mathrm{CD}}$, última coluna mostra o resultado $\mathrm{T}$ (true). Isso significa que se a tensão de escoamento for menor que a tensão, os cálculos podem ser considerados válidos. Caso contrário, deverão ser descartados porque as equações que envolvem o módulo de elasticidade não podem ser mais utilizadas.

Quadro 2 - Valores das forças $F_{C D}$ e $F_{A B}$ obtidos pela simulação no Scilab.

\begin{tabular}{|c|c|c|}
\hline Material & $\mathrm{F}_{\mathrm{CD}}[\mathrm{kN}]$ & $\mathrm{F}_{\mathrm{AB}}[\mathrm{kN}]$ \\
\hline Aço estrutural (ASTM-A36) & 90 & -60 \\
\hline $\begin{array}{c}\text { Aço baixa liga e alta resistência (ASTM- } \\
\text { A709 Classe 345) }\end{array}$ & 90 & -60 \\
\hline $\begin{array}{c}\text { Aço baixa liga e alta resistência (ASTM- } \\
\text { A913 Classe 450) }\end{array}$ & 90 & -60 \\
\hline $\begin{array}{c}\text { Aço baixa liga e alta resistência (ASTM- } \\
\text { A992 Classe 345) }\end{array}$ & 90 & -60 \\
\hline Aço temperado e revenido ASTM- \\
A709 CLASSE 690 & 90 & -60 \\
\hline Aço inoxidável AISI 302 laminado a frio & 90 & -60 \\
\hline Aço inoxidável AISI 302 recozido & 90 & -60 \\
\hline Aço de reforço média resistência & 90 & -60 \\
\hline Aço de reforço alta resistência & 90 & -60 \\
\hline Ferro fundido cinzento 4,5\% C, ASTM \\
A-48
\end{tabular}




\begin{tabular}{|c|c|c|}
\hline Alumínio liga 1100-H14 (99\% Al) & 90 & -60 \\
\hline Alumínio liga 2012-T6 & 90 & -60 \\
\hline Alumínio liga 2024-T4 & 90 & -60 \\
\hline Alumínio liga 5456-H116 & 90 & -60 \\
\hline Alumínio liga 6061-T6 & 90 & -60 \\
\hline Alumínio liga 7075-T6 & 90 & -60 \\
\hline $\begin{array}{l}\text { Cobre livre de oxigênio }(99,9 \% \mathrm{Cu}) \\
\text { recozido }\end{array}$ & 90 & -60 \\
\hline $\begin{array}{l}\text { Cobre livre de oxigênio }(99,9 \% \mathrm{Cu}) \\
\text { trefilado a frio }\end{array}$ & 90 & -60 \\
\hline $\begin{array}{l}\text { Latão amarelo ( } 65 \% \mathrm{Cu}, 35 \% \mathrm{Zn}) \\
\text { laminado a frio }\end{array}$ & 90 & -60 \\
\hline $\begin{array}{l}\text { Latão amarelo }(65 \% \mathrm{Cu}, 35 \% \mathrm{Zn}) \\
\text { recozido }\end{array}$ & 90 & -60 \\
\hline $\begin{array}{l}\text { Latão vermelho ( } 85 \% \mathrm{Cu}, 15 \% \mathrm{Zn}) \\
\text { laminado a frio }\end{array}$ & 90 & -60 \\
\hline $\begin{array}{l}\text { Latão vermelho }(85 \% \mathrm{Cu}, 15 \% \mathrm{Zn}) \\
\text { recozido }\end{array}$ & 90 & -60 \\
\hline $\begin{array}{l}\text { Liga bronze + estanho }(88 \% \mathrm{Cu}, 8 \% \mathrm{Sn} \text {, } \\
\qquad 4 \% \mathrm{Zn})\end{array}$ & 90 & -60 \\
\hline $\begin{array}{c}\text { Liga bronze + manganês (63\% Cu, 25\% } \\
\text { Zn, } 6 \% \mathrm{Al}, 3 \% \mathrm{Mn}, 3 \% \mathrm{Fe})\end{array}$ & 90 & -60 \\
\hline $\begin{array}{l}\text { Liga bronze + alumínio ( } 81 \% \mathrm{Cu}, 4 \% \mathrm{Ni} \text {, } \\
\qquad 4 \% \mathrm{Fe}, 11 \% \mathrm{Al})\end{array}$ & 90 & -60 \\
\hline
\end{tabular}

Quadro 3 - Comparação entre a tensão de escoamento $\sigma_{\mathrm{eCD}}$ e a tensão $\sigma_{\mathrm{CD}}$, sendo que o Scilab informa como $T$ (true) se $\sigma_{\mathrm{eCD}}<\sigma_{\mathrm{CD}}$, caso contrário como $F$ ( false).

\begin{tabular}{|c|c|c|c|}
\hline Material & $\sigma_{\mathrm{eCD}}[\mathrm{MPa}]$ & $\sigma_{\mathrm{CD}}[\mathrm{MPa}]$ & Comparacao \\
\hline $\begin{array}{c}\text { Aço estrutural (ASTM-A36) } \\
\text { Aço baixa liga e alta resistência (ASTM- } \\
\text { A709 Classe 345) }\end{array}$ & 250 & 150 & $\mathrm{~T}$ \\
\hline $\begin{array}{c}\text { Aço baixa liga e alta resistência (ASTM- } \\
\text { A913 Classe 450) }\end{array}$ & 0 & 150 & $\mathrm{~F}$ \\
\hline $\begin{array}{c}\text { Aço baixa liga e alta resistência (ASTM- } \\
\text { A992 Classe 345) }\end{array}$ & 0 & 150 & $\mathrm{~F}$ \\
\hline Aço temperado e revenido ASTM- \\
A709 CLASSE 690
\end{tabular}




\begin{tabular}{|c|c|c|c|}
\hline Aço inoxidável AISI 302 recozido & 0 & 150 & $\mathrm{~F}$ \\
\hline Aço de reforço média resistência & 77 & 150 & $\mathrm{~F}$ \\
\hline Aço de reforço alta resistência & 77 & 150 & $\mathrm{~F}$ \\
\hline \multicolumn{4}{|l|}{ Ferro fundido cinzento 4,5\% C, ASTM } \\
\hline A-48 & 0 & 150 & $\mathrm{~F}$ \\
\hline \multicolumn{4}{|l|}{ Ferro fundido maleável 2\% C, 1\% Si, } \\
\hline ASTM A-47 & 230 & 150 & $\mathrm{~T}$ \\
\hline Alumínio liga 1100-H14 (99\% Al) & 95 & 150 & $\mathrm{~F}$ \\
\hline Alumínio liga 2012-T6 & 400 & 150 & $\mathrm{~T}$ \\
\hline Alumínio liga 2024-T4 & 325 & 150 & $\mathrm{~T}$ \\
\hline Alumínio liga 5456-H116 & 230 & 150 & $\mathrm{~T}$ \\
\hline Alumínio liga 6061-T6 & 240 & 150 & $\mathrm{~T}$ \\
\hline Alumínio liga 7075-T6 & 500 & 150 & $\mathrm{~T}$ \\
\hline \multicolumn{4}{|l|}{ Cobre livre de oxigênio $(99,9 \% \mathrm{Cu})$} \\
\hline recozido & 70 & 150 & $\mathrm{~F}$ \\
\hline \multicolumn{4}{|l|}{ Cobre livre de oxigênio $(99,9 \% \mathrm{Cu})$} \\
\hline trefilado a frio & 265 & 150 & $\mathrm{~T}$ \\
\hline \multicolumn{4}{|l|}{ Latão amarelo (65\% Cu, 35\% Zn) } \\
\hline laminado a frio & 410 & 150 & $\mathrm{~T}$ \\
\hline \multicolumn{4}{|l|}{ Latão amarelo (65\% Cu, 35\% Zn) } \\
\hline recozido & 100 & 150 & $\mathrm{~F}$ \\
\hline \multicolumn{4}{|l|}{ Latão vermelho (85\% Cu, 15\% Zn) } \\
\hline laminado a frio & 435 & 150 & $\mathrm{~T}$ \\
\hline \multicolumn{4}{|l|}{ Latão vermelho (85\% Cu, 15\% Zn) } \\
\hline recozido & 70 & 150 & $\mathrm{~F}$ \\
\hline \multicolumn{4}{|l|}{ Liga bronze + estanho (88\% Cu, $8 \%$ Sn, } \\
\hline $4 \% \mathrm{Zn})$ & 145 & 150 & $\mathrm{~F}$ \\
\hline \multicolumn{4}{|l|}{ Liga bronze + manganês (63\% Cu, 25\% } \\
\hline Zn, 6\% Al, 3\% Mn, 3\% Fe) & 330 & 150 & $\mathrm{~T}$ \\
\hline Liga bronze + alumínio (81\% Cu, 4\% Ni, & & & \\
\hline $4 \% \mathrm{Fe}, 11 \% \mathrm{Al})$ & 275 & 150 & $\mathrm{~T}$ \\
\hline
\end{tabular}

O quadro 4 mostra os valores de deslocamento $\delta_{B}, \delta_{D}, x$ e $\delta_{E}$ em milímetros. Os valores serão considerados somente se $\sigma_{\mathrm{eCD}}<\sigma_{\mathrm{CD}}$. Dessa forma, na coluna "Comparação" onde o resultado é $\mathrm{F}$ (false), os valores de deslocamento serão ignorados e as linhas foram marcadas em vermelho. Caso contrário, os valores aceitos possuem $\mathrm{T}$ (true), nessa mesma coluna, e as linhas estão em branco. 
Quadro 4 - Valores obtidos dos deslocamentos $\delta_{B}, \delta_{D}, x$ e $\delta_{E}$ em $\mathrm{mm}$ válidos somente para as linhas em branco.

\begin{tabular}{|c|c|c|c|c|c|}
\hline Material & $\delta_{B}[\mathrm{~mm}]$ & $\delta_{D}[\mathrm{~mm}]$ & $\mathrm{x}[\mathrm{mm}]$ & $\delta_{E}[\mathrm{~mm}]$ & Comparação \\
\hline Aço estrutural (ASTM-A36) & $-0,51$ & 0,3 & 73,7 & 1,93 & $\mathrm{~T}$ \\
\hline $\begin{array}{c}\text { Aço baixa liga e alta resistência } \\
\text { (ASTM-A709 Classe 345) }\end{array}$ & $-0,51$ & 0,3 & 73,7 & 1,93 & $\mathrm{~F}$ \\
\hline $\begin{array}{c}\text { Aço baixa liga e alta resistência } \\
\text { (ASTM-A913 Classe 450) }\end{array}$ & $-0,51$ & 0,3 & 73,7 & 1,93 & $\mathrm{~F}$ \\
\hline $\begin{array}{c}\text { Aço baixa liga e alta resistência } \\
\text { (ASTM-A992 Classe 345) }\end{array}$ & $-0,51$ & 0,3 & 73,7 & 1,93 & $\mathrm{~F}$ \\
\hline $\begin{array}{l}\text { Aço temperado e revenido ASTM- } \\
\text { A709 CLASSE } 690\end{array}$ & $-0,51$ & 0,3 & 73,7 & 1,93 & $\mathrm{~F}$ \\
\hline $\begin{array}{c}\text { Aço inoxidável AISI } 302 \text { laminado } \\
\text { a frio }\end{array}$ & $-0,51$ & 0,32 & 76,1 & 1,98 & $\mathrm{~F}$ \\
\hline Aço inoxidável AISI 302 recozido & $-0,51$ & 0,32 & 76,1 & 1,98 & $\mathrm{~F}$ \\
\hline Aço de reforço média resistência & $-0,51$ & Inf & Nan & Nan & $\mathrm{F}$ \\
\hline Aço de reforço alta resistência & $-0,51$ & Inf & Nan & Nan & $\mathrm{F}$ \\
\hline $\begin{array}{l}\text { Ferro fundido cinzento } 4,5 \% \text { C, } \\
\text { ASTM A-48 }\end{array}$ & $-0,51$ & 0,87 & 126 & 3,64 & $\mathrm{~F}$ \\
\hline $\begin{array}{l}\text { Ferro fundido maleável 2\% C, } 1 \% \\
\qquad \text { Si, ASTM A-47 }\end{array}$ & $-0,51$ & 0,36 & 82,8 & 2,12 & $\mathrm{~T}$ \\
\hline Alumínio liga 1100-H14 (99\% Al) & $-0,51$ & 0,86 & 125 & 3,6 & $\mathrm{~F}$ \\
\hline Alumínio liga 2012-T6 & $-0,51$ & 0,8 & 122 & 3,43 & $\mathrm{~T}$ \\
\hline Alumínio liga 2024-T4 & $-0,51$ & 0,82 & 123 & 3,49 & $\mathrm{~T}$ \\
\hline Alumínio liga 5456-H116 & $-0,51$ & 0,83 & 124 & 3,53 & $\mathrm{~T}$ \\
\hline Alumínio liga 6061-T6 & $-0,51$ & 0,86 & 125 & 3,6 & $\mathrm{~T}$ \\
\hline Alumínio liga 7075-T6 & $-0,51$ & 0,83 & 124 & 3,53 & $\mathrm{~T}$ \\
\hline $\begin{array}{l}\text { Cobre livre de oxigênio }(99,9 \% \\
\qquad \text { Cu) recozido }\end{array}$ & $-0,51$ & 0,5 & 98,6 & 2,53 & $\mathrm{~F}$ \\
\hline $\begin{array}{l}\text { Cobre livre de oxigênio }(99,9 \% \\
\qquad \text { Cu) trefilado a frio }\end{array}$ & $-0,51$ & 0,5 & 98,6 & 2,53 & $\mathrm{~T}$ \\
\hline $\begin{array}{l}\text { Latão amarelo (65\% Cu, 35\% Zn) } \\
\text { laminado a frio }\end{array}$ & $-0,51$ & 0,57 & 105 & 2,74 & $\mathrm{~T}$ \\
\hline $\begin{array}{l}\text { Latão amarelo }(65 \% \text { Cu, 35\% Zn) } \\
\text { recozido }\end{array}$ & $-0,51$ & 0,57 & 105 & 2,74 & $\mathrm{~F}$ \\
\hline $\begin{array}{l}\text { Latão vermelho ( } 85 \% \mathrm{Cu}, 15 \% \mathrm{Zn}) \\
\text { laminado a frio }\end{array}$ & $-0,51$ & 0,5 & 98,6 & 2,53 & $\mathrm{~T}$ \\
\hline $\begin{array}{l}\text { Latão vermelho }(85 \% \mathrm{Cu}, 15 \% \mathrm{Zn}) \\
\text { recozido }\end{array}$ & $-0,51$ & 0,5 & 98,6 & 2,53 & $\mathrm{~F}$ \\
\hline
\end{tabular}

Revista Univap - revista.univap.br

São José dos Campos-SP-Brasil, v. 25, n. 47, jul. 2019. ISSN 2237-1753 


\begin{tabular}{|c|c|c|c|c|c|}
\hline $\begin{array}{c}\text { Liga bronze + estanho (88\% Cu, } \\
8 \% \text { Sn, 4\% Zn) }\end{array}$ & $-0,51$ & 0,63 & 110 & 2,92 & $\mathrm{~F}$ \\
\hline $\begin{array}{c}\text { Liga bronze + manganês (63\% Cu, } \\
25 \% \text { Zn, 6\% Al, 3\% Mn, 3\% Fe) }\end{array}$ & $-0,51$ & 0,57 & 105 & 2,74 & $\mathrm{~T}$ \\
\hline $\begin{array}{c}\text { Liga bronze + alumínio (81\% Cu, } \\
4 \% \mathrm{Ni}, 4 \% \mathrm{Fe}, 11 \% \mathrm{Al})\end{array}$ & $-0,51$ & 0,55 & 103 & 2,66 & $\mathrm{~T}$ \\
\hline
\end{tabular}

\section{O código do programa é apresentado a seguir:}

clear

$\mathrm{xBD}=0.2$

$\mathrm{xDE}=0.4$

$\mathrm{yAB}=0.3$;

$\mathrm{yBC}=0.4$

$\mathrm{EAB}=70^{*} 10^{\wedge} 9$;

$\mathrm{AAB}=500^{*} 10^{\wedge}-6$;

$\mathrm{ACD}=600 * 10^{\wedge}-6$

$\mathrm{PE}=30^{*} 10^{\wedge} 3$;

FCDkNfinal=[];

FABkNfinal $=[]$

xmmfinal $=[]$;

$\delta$ Emmfinal $=[]$

$\sigma$ CDfinal $=[]$;

comparacaofinal $=[]$

$\delta$ Bfinal=[];

$\delta$ Dfinal $=[] ;$

$\mathrm{i}=1$;

$\sigma \mathrm{eCD}=[410 ; 250 ; 0 ; 0 ; 0 ; 0 ; 0 ; 0 ; 77 ; 77 ; 0 ; 230 ; 95 ; 400 ; 325 ; 230 ; 240 ; 500 ; 70 ; 265 ; 410 ; 100 ; 435 ; 70 ; 145 ; 330 ; 275]^{*} 10^{\wedge} 6$; Material= ['Exercicio';'Aço estrutural (ASTM-A36)';'Aço baixa liga e alta resistência (ASTM-A709 Classe 345)';'Aço baixa liga e alta resistência (ASTM-A913 Classe 450)';'Aço baixa liga e alta resistência (ASTM-A992 Classe 345)';'Aço temperado e revenido ASTM-A709 CLASSE 690'; 'Aço inoxidável AISI 302 laminado a frio';'Aço inoxidável AISI 302 recozido';'Aço de reforço média resistência';'Aço de reforço alta resistência'; 'Ferro fundido cinzento 4,5\% C, ASTM A48';'Ferro fundido maleável 2\% C, 1\% Si, ASTM A-47';'Alumínio liga 1100-H14 (99\% Al)';'Alumínio liga 2012-

T6';'Alumínio liga 2024-T4';'Alumínio liga 5456-H116';'Alumínio liga 6061-T6';'Alumínio liga 7075-T6';'Cobre livre de oxigênio $(99,9 \% \mathrm{Cu}$ ) recozido';'Cobre livre de oxigênio $(99,9 \% \mathrm{Cu}$ ) trefilado a frio';'Latão amarelo $(65 \% \mathrm{Cu}$, 35\% $\mathrm{Zn})$ laminado a frio';'Latão amarelo $(65 \% \mathrm{Cu}, 35 \% \mathrm{Zn})$ recozido';'Latão vermelho (85\% Cu, $15 \% \mathrm{Zn}$ ) laminado a frio';'Latão vermelho (85\% Cu, 15\% Zn) recozido';'Liga bronze + estanho (88\% Cu, 8\% Sn, 4\% Zn)';'Liga bronze + manganês $(63 \% \mathrm{Cu}, 25 \% \mathrm{Zn}, 6 \% \mathrm{Al}, 3 \% \mathrm{Mn}, 3 \% \mathrm{Fe})$ ';'Liga bronze + alumínio (81\% Cu, 4\% Ni, 4\% Fe, $11 \% \mathrm{Al}$ )']; for

$\mathrm{ECD}=[200,200,200,200,200,200,190,190,0,0,69,165,70,75,73,72,70,72,120,120,105,105,120,120,95,105,110]^{*} 10^{\wedge} 9$; $\mathrm{FCD}=\mathrm{PE} *(\mathrm{xBD}+\mathrm{xDE}) / \mathrm{xBD}$;

$\mathrm{FAB}=-\mathrm{PE} *(\mathrm{xDE} / \mathrm{XBD})$;

$\delta \mathrm{B}=\left(\mathrm{FAB}^{*} \mathrm{yAB}\right) /\left(\mathrm{AAB}^{*} \mathrm{EAB}\right) ;$

$\delta \mathrm{D}=(\mathrm{FCD} * \mathrm{yBC}) /(\mathrm{ACD} * \mathrm{ECD}) ;$

$\mathrm{x}=\left(\delta \mathrm{D}^{*} \mathrm{xBD}\right) /(\delta \mathrm{D}-\delta \mathrm{B})$;

$\delta \mathrm{E}=\delta \mathrm{D}^{*}(\mathrm{xDE}+\mathrm{x}) / \mathrm{x}$;

$\sigma \mathrm{CD}=\mathrm{FCD} / \mathrm{ACD}$;

comparacao $=\sigma \mathrm{CD}<\sigma \mathrm{eCD}(\mathrm{i})$;

format(5)

$\mathrm{FCDkN}=\mathrm{FCD}^{*} 10^{\wedge}-3$;

$\mathrm{FABkN}=\mathrm{FAB}^{*} 10^{\wedge}-3$;

$\mathrm{xmm}=\mathrm{x}^{*} 10^{\wedge} 3$;

$\delta \mathrm{Emm}=\delta \mathrm{E}^{*} 10^{\wedge} 3$

FCDkNfinal=[FCDkNfinal;FCDkN];

FABkNfinal=[FABkNfinal;FABkN];

xmmfinal $=[$ xmmfinal; $x m m]$;

$\delta$ Emmfinal $=[\delta$ Emmfinal; $\delta$ Emm $]$;

$\sigma \mathrm{CD}$ final $=[\sigma \mathrm{CD}$ final; $\sigma \mathrm{CD}]$;

comparacaofinal=[comparacaofinal;comparacao];

$\delta$ Bfinal $=[\delta$ Bfinal $; \delta \mathrm{B}]$;

$\delta$ Dfinal $=[\delta$ Dfinal; $\delta \mathrm{D}]$;

$\mathrm{i}=\mathrm{i}+1$;

end 


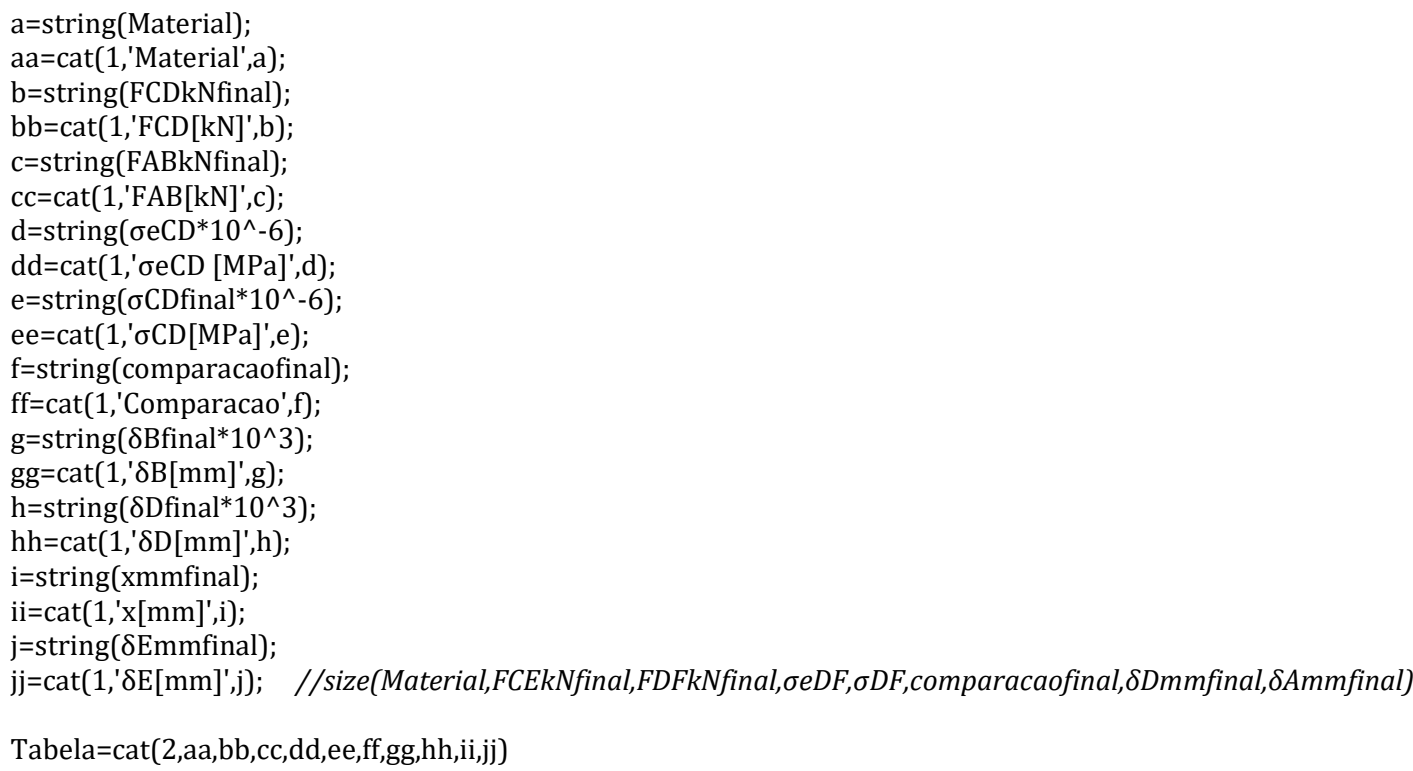

\section{CONCLUSÃO}

Devido a se optar por um sistema mecânico que opere no regime elástico, a equação 4 só poderá ser aplicada se a tensão calculada para o cilindro CD for inferior a tensão de escoamento, isto é, $\sigma_{\mathrm{eCD}}<\sigma_{\mathrm{CD}}$. Dessa forma, quando a tensão $\sigma_{\mathrm{CD}}$ for superior a $\sigma_{\mathrm{eCD}}$, os valores de deslocamento obtidos pelo software Scilab deverão ser desprezados. De acordo com a tabela 4 , as linhas em vermelho referem-se aos valores que deverão ser ignorados.

Os materiais que apresentaram a tensão inferior a tensão de escoamento $\left(\sigma_{\mathrm{eCD}}<\right.$ $\sigma_{\mathrm{CD}}$ ), para a carga axial de $30 \mathrm{kN}$, foram: aço estrutural (ASTM-A36), ferro fundido maleável 2\% C, 1\% Si, ASTM A-47, alumínio liga 2012-T6, alumínio liga 2024-T4, alumínio liga 5456-H116,alumínio liga 6061-T6, alumínio liga 7075-T6, cobre livre de oxigênio $(99,9 \% \mathrm{Cu})$ trefilado a frio, latão amarelo $(65 \% \mathrm{Cu}, 35 \% \mathrm{Zn})$ laminado a frio, latão vermelho $(85 \% \mathrm{Cu}, 15 \% \mathrm{Zn})$ laminado a frio, liga bronze + manganês $(63 \% \mathrm{Cu}$, $25 \% \mathrm{Zn}, 6 \% \mathrm{Al}, 3 \% \mathrm{Mn}, 3 \% \mathrm{Fe})$, liga bronze + alumínio (81\% Cu, 4\% Ni, 4\% Fe, 11\% $\mathrm{Al})$. Dessa forma, esses materiais tiveram os seus valores de deslocamento calculados $\delta_{B}, \delta_{D}$ e $\delta_{E}$.

Os maiores valores de deslocamento $\delta_{D}$ e $\delta_{E}$, calculados via Scilab, foi para 0 alumínio liga 6061-T6: $\delta_{D}=0,86 \mathrm{~mm}$ e $\delta_{E}=125 \mathrm{~mm}$. Estruturas cilíndricas com esse tipo de material não resistem muito a forças de $30 \mathrm{kN}$, quando comparado aos materiais listados da tabela 01.

O material mais resistente ao carregamento de $30 \mathrm{kN}$ foi o aço estrutural (ASTMA36), apresentando $\delta_{D}=0,3 \mathrm{~mm}$ e $\delta_{E}=73,7 \mathrm{~mm}$.

Dessa forma, conclui-se que o cilindro CD constituído de alumínio liga 6061-T6 faz com que haja maior deslocamento da barra rígida $B D E$, enquanto se utilizado o aço estrutural (ASTM-A36), o deslocamento seria inferior. 
Materiais em que a tensão calculada foi superior à tensão de escoamento informada na tabela 1 devem ter os valores $\delta_{B}$, $\delta_{D}$ e $\delta_{E}$ ignorados porque o escoamento não esteve no regime elástico. Esses materiais foram: aço baixa liga e alta resistência (ASTM-A709 Classe 345), aço baixa liga e alta resistência (ASTM-A913 Classe 450, aço baixa liga e alta resistência (ASTM-A992 Classe 345, aço temperado e revenido ASTM-A709 CLASSE 69, aço inoxidável AISI 302 laminado a frio, aço inoxidável AISI 302 recozido, aço de reforço média resistência, aço de reforço alta resistência, ferro fundido cinzento 4,5\% C, ASTM A-48, alumínio liga 1100-H14 (99\% Al), cobre livre de oxigênio $(99,9 \% \mathrm{Cu})$ recozido,latão amarelo $(65 \% \mathrm{Cu}, 35 \% \mathrm{Zn})$ recozido, latão vermelho $(85 \% \mathrm{Cu}, 15 \% \mathrm{Zn})$ recozido, liga bronze + estanho (88\% Cu, $8 \% \mathrm{Sn}, 4 \% \mathrm{Zn})$.

\section{REFERÊNCIAS}

BEER, F. P. et.al. Mecânica dos materiais. 5.ed. Rio de Janeiro: Mc Graw Hill, 2008.

DE OLIVEIRA, A. M.; DE OLIVEIRA, A. M. N.; BUENO, R. C. Scilab: uma abordagem prática aplicada a problemas reais da engenheira. São Paulo: Laboratório Maxwell, 2016.

HIBBELER, R. C. Estática: mecânica para engenharia. 10. ed. São Paulo: Pearson Prentice Hall, 2005.

LEET, K. M.; UANG, C. M.; GILBERT A. M. Fundamentos da análise estrutural. 3. ed. Porto Alegre: AMGH, 2010.

MERIAM, J. L.; KRAIGE, L. G. Mecânica para engenharia. 6. ed. São Paulo: LTC, 2009. v. 1.

NASH, W. A.; POTTER, M. C. Resistência dos Materiais. 5. ed. São Paulo: Bookman, 2014. 